\title{
Preferential Location of Germanium in the UTL and IPC-2a Zeolites
}

Samuel O. Odoh ${ }^{1}$, Michael W. Deem ${ }^{*, 2,}$ and Laura Gagliardi*,1

1) Department of Chemistry, Chemical Theory Center, and Supercomputing Institute, University of Minnesota, Minneapolis, Minnesota 55455-0431, United States

2) Department of Bioengineering and Department of Physics \& Astronomy, Rice University, Houston, Texas 77005, United States

\begin{abstract}
The preferential location of $\mathrm{Ge}$ in the double four-membered rings (D4R) of zeolite UTL has been examined with periodic density functional theory calculations. In silicate UTL, Ge atoms are preferentially sited at the T1 and T2 atoms in the D4R cages. The preference for siting Ge atoms at the D4R sites over other $\mathrm{T}$ atom sites in UTL increases as more $\mathrm{Si}$ atoms are replaced by Ge. Our calculations show that at least $3 \mathrm{Ge}$ atoms can be located in the D4R cages before any appreciable occupancy at non-D4R sites. Comparison of Ge atom siting in UTL to zeolite IPC-2a shows that the single four-membered ring (S4R) sites in the latter are not the most favourable sites.
\end{abstract}

\section{INTRODUCTION}

Zeolite UTL $^{1}$ (IM-12, ITQ-15) is a germanosilicate zeolite that was synthesized by Corma et al. ${ }^{2}$ and Paillaud et al. ${ }^{3}$ It has a two-dimensional (2D) pore channel system with large pores formed by intersecting 14- and 12-ring channels that are supported by double four-ring (D4R) units, Figure 1. Ge atoms are preferentially located at the $\mathrm{T}$ atom sites in the D4R cages. We note that the tetrahedrally coordinated framework atoms in zeolites, 
Si and Ge in the case of germanosilicate UTL, are labelled as T atoms. Recently, Čejka et al. ${ }^{4}$ reported the synthesis and characterization of two novel zeolites with targeted topologies, IPC-2a and IPC-4, by replacing the germanium-rich D4R units in the UTL zeolite with single four-ring (S4R) cages and oxygen atoms respectively, Figures 2 and 3. The protocol used in their experiments, Assembly, Disassembly, Organization and Reassembly (ADOR), suggests that the layers of UTL are initially separated from its D4R cages before the latter are replaced by S4R cages or oxygen atoms in a reassembly process. ${ }^{4}$ An advantage of the ADOR protocol or certainly its underpinning idea is that it is modular and can in theory be applied to other zeolites containing D4R cages. This experimental procedure provides a pathway for developing zeolite frameworks with specific or targeted pore sizes by choosing the units and linkers to replace the D4R cages. Replacement of the D4R cages by larger units will lead to

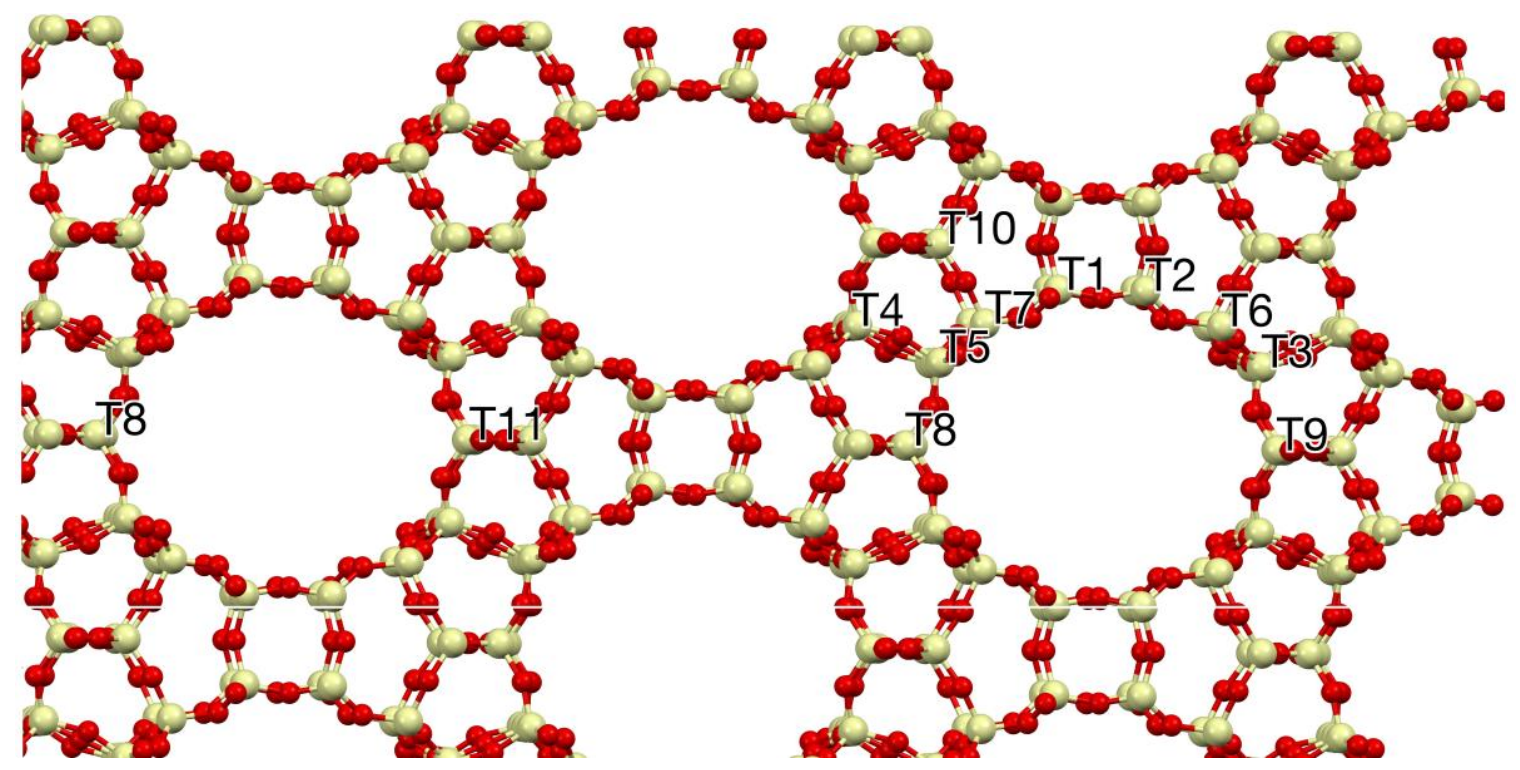

Figure 1: The positions of unique $\mathrm{T}$ atoms in silicate $\mathrm{UTL}$. The $\mathrm{Si}$ and $\mathrm{O}$ atoms are shown in yellow and red respectively. The D4R cages contain the T1 and T2 atoms. 


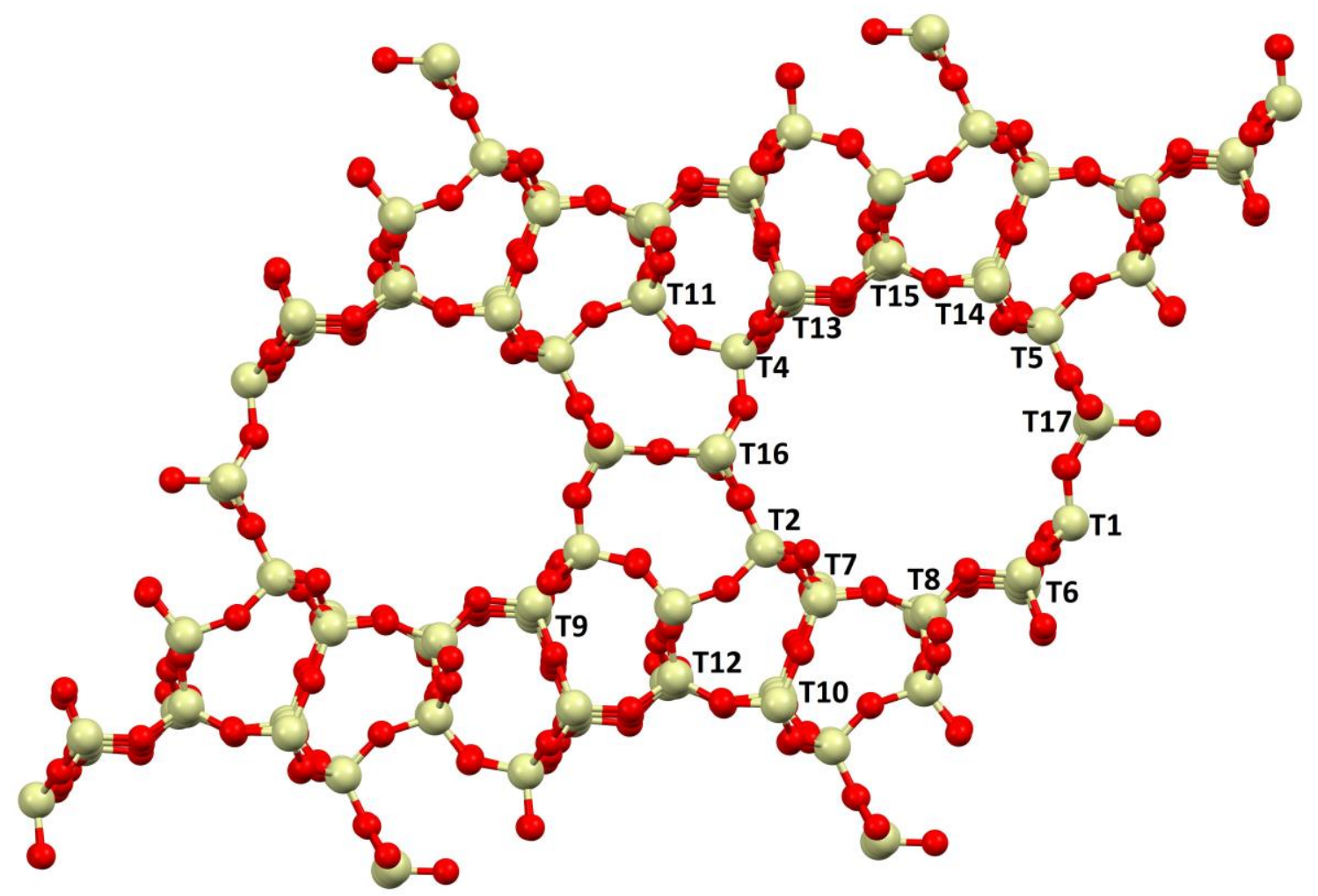

Figure 2: The $\mathrm{T}$ atom sites in silicate IPC-2a. The $\mathrm{Si}$ and $\mathrm{O}$ atoms are shown in yellow and red respectively. The S4R cages contain the T16 and T17 atoms.

zeolites with larger pore channels while replacement with smaller units will lead to smaller pore channels. This specific targeting of pore sizes is of great importance to the potential of zeolites for use in shape/size-selective catalysis, gas adsorption and gas storage.

It is however crucial to note that the ADOR protocol is underpinned by the knowledge that the Ge atoms of germanosilicate UTL are preferentially sited in the D4R cages. This preferential siting allowed Čejka et al. ${ }^{4}$ to develop a procedure to separate the layers of UTL from its Ge-rich D4R cages prior to reassembly. The preferential location of Ge in the D4R cages of several silicate zeolites has been known for quite some time. Indeed, it 


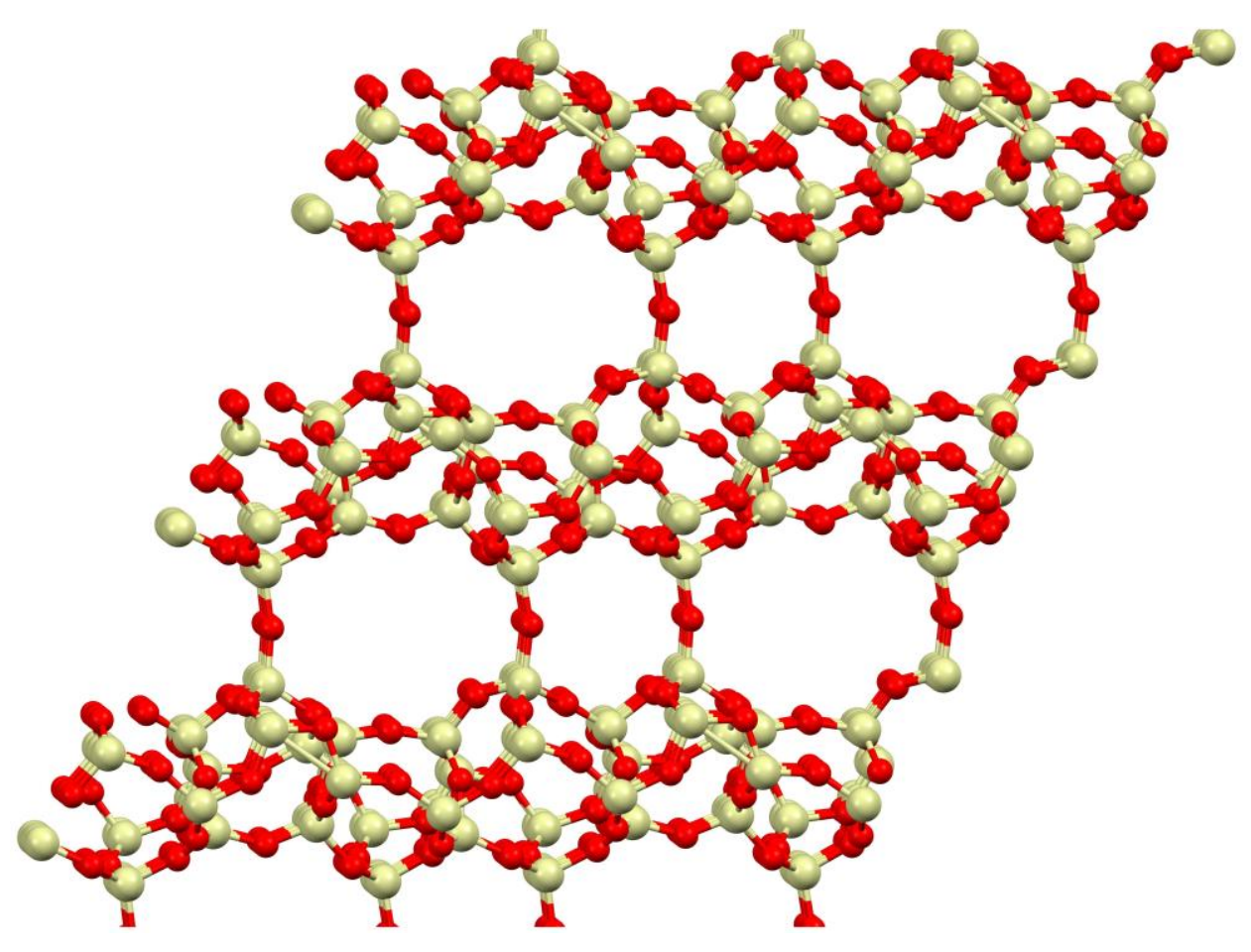

Figure 3: The unit-cell of the silicate IPC-4 zeolite. ${ }^{4}$ The $\mathrm{Si}$ and $\mathrm{O}$ atoms are shown in yellow and red respectively. The layers are connected by bridging oxygen atoms.

has been shown that the presence of Ge can actually direct the zeolite synthesis towards frameworks containing D4R cages. ${ }^{5}$ Also, the syntheses of some zeolites with D4R cages are feasible only in the presence of Ge. ${ }^{5-6}$ The ADOR process as well as the decrease in the concentration of $\mathrm{Ge}$ in the resulting zeolites has been additionally described in recent topical reviews by Roth et al. ${ }^{7}$ This preferential siting of Ge in the D4R cages is however not the only case of preferential location of metal or metalloid atoms in zeolite frameworks. As an example, it is known that zinc $(\mathrm{Zn})$ is preferentially sited in the threemembered rings (3R) in zeolite VNI (VPI-9) ${ }^{8}$ and encourages the synthesis of zeolite VET (VPI-8). ${ }^{9}$

There have been several attempts to rationalize the preferential location of Ge in the D4R clusters of silicate zeolites. O'Keeffe and Yaghi found that the Ge-O-Ge angles present in 
germanate zeolites are lower than analogous Si-O-Si angles in silicate zeolites. ${ }^{10}$ Sastre et al. rationalized that during Ge substitution in silicate zeolites, the Ge atoms would be located in parts of the zeolite framework conducive for lower T-O-T (where T represents $\mathrm{Si}$ or Ge atoms) angles. ${ }^{11}$ They subsequently used atomistic force fields and a $\mathrm{Si} / \mathrm{Ge}$ potential to study the preferential localization of Ge in the D4R cages of the AST, ASV, BEA, BEC, ISV and LTA zeolites. ${ }^{1}$ Although, they found that tetrahedral positions with lower $\mathrm{Si}-\mathrm{O}-\mathrm{Ge}$ angles are indeed energetically favoured for $\mathrm{Ge}$ atom siting, their results do not fully explain the preferential siting in the D4R cages as other positions outside the D4R cages with low Si-O-Ge angles were also found to be low in energy. Zwijnenburg et al. carried out density functional theory (DFT) calculations on model polyhedral clusters with varying amounts of Ge substituents. ${ }^{12}$ They noted that Ge atoms reduce the destabilization of the D4R cluster cages with respect to other types of polyhedral cages. The stabilization energy amounts to $0.3-0.5 \mathrm{~kJ} / \mathrm{mol}$ when $\mathrm{Si}$ atoms are replaced by $\mathrm{Ge}$ atoms (labeled as $\mathrm{Si} / \mathrm{Ge}$ substitution). These energies are however too small to adequately explain the macroscopic observation of the preferential location of Ge atoms in the D4R cages. They are much smaller than the errors associated with approximate DFT, making it impossible to draw any conclusions based on these numbers. Given the outcome of the previous force field ${ }^{11}$ and cluster model calculations ${ }^{12}$, and the importance of the D4R cages to the ADOR process ${ }^{4}$, there is a need to computationally predict the preferential siting of Ge in the D4R structures of silicate zeolites in comparison to other $\mathrm{T}$ atom positions as well as the energetic stabilities associated with this preferential siting. A rational explanation for the preferential siting of Ge in the D4R cages is also desirable. Periodic DFT is a suitable approach for studying the preferential location of $\mathrm{Ge}$ in the 
D4R cages of UTL as Kamakoti et al. ${ }^{13}$ have previously shown that calculations with DFT can adequately reproduce preferential siting of Ge at the D4R sites of the BEC ${ }^{14}$ zeolite. However, the BEC zeolite contains only three crystallographically unique Tsites $^{14}$ and therefore lacks the complexity of structural behavior found in UTL, which has twelve unique T-atom sites, Figure 1.

In this work, we have investigated the preferential location of Ge atoms in UTL with periodic DFT calculations. The primary motivations for this study are two-fold. It is hoped that periodic calculations at the DFT level can provide a more definitive explanation of the preferential location of Ge in zeolites with D4R cages. Theoretical calculations at this level, in contrast to calculations using cluster models or force fields and $\mathrm{Si} / \mathrm{Ge}$ potentials, have allowed for a quantitative determination of the relative substitution energies associated with the preferential siting of Ge in UTL. Secondly, we have compared the energetics associated with $\mathrm{Si} / \mathrm{Ge}$ substitutions in UTL to those in IPC2a. Through this comparison, we show that the magnitude of the average T-O-T angles centred at each site is not the sole determinant of the preference for Ge localization.

\section{Computational Details}

All the DFT calculations in this work were carried out with the VASP 5.3.3 ${ }^{15}$ package, which solves the equations of approximate Kohn-Sham DFT with periodic boundary conditions while using a plane-wave basis set formalism. The electron-ion interaction in these calculations is described with the projector augmented wave (PAW) method developed by Blöchl ${ }^{16}$ with an energy cutoff of $550 \mathrm{eV}$ while employing the $\mathrm{PBE}^{17}$ functional. This density functional and plane waves basis set scheme was used for full optimization of the structures and lattices of UTL and IPC-2a until the forces on all the 
atoms were smaller than $0.02 \mathrm{eV} / \mathrm{A}$. Dispersion effects ${ }^{18}$ were included by systematic optimization of all the structures of UTL and IPC-2a with the vdW-DF2 $2^{19}$ and optPBE ${ }^{20}$ functionals. The sampling of the Brillouin-zone of UTL is restricted to the gamma $(\Gamma)$ point while the sampling of IPC-2a was carried out with a $3 \times 3 \times 3$ Monkhorst-Pack kpoint mesh. Regarding the suitability of the PBE, vdW-DF2 and optPBE functionals for studying the energetic ordering of available sites for locating Ge in UTL, we note that these functionals predict the relative stabilities of $\alpha$-cristobalite polymorph of silica ${ }^{21}$ relative to the $\alpha$-quartz polymorph as $3.4,3.8$ and $3.4 \mathrm{~kJ} / \mathrm{mol}$ respectively. These values in are good agreement with the experimental enthalpy of $2.8 \pm 2.2 \mathrm{~kJ} / \mathrm{mol}$ obtained by Petrovic et al. ${ }^{22}$ or $2.6 \mathrm{~kJ} / \mathrm{mol}$ reported by Saxena et al. ${ }^{23}$ Kamakoti et al. ${ }^{13}$ and Catti et al. ${ }^{24}$ previously reported an energy difference of 4.6 and $9.3 \mathrm{~kJ} / \mathrm{mol}$ respectively between the $\alpha$-cristobalite and $\alpha$-quartz polymorphs with the local density approximation ${ }^{25}$ (LDA) exchange-correlation functional.

The preferential location of Ge atoms in UTL and IPC-2a was studied by carrying out geometry optimizations for all the mixed $\mathrm{Si} / \mathrm{Ge}$ zeolites formed by replacing each $\mathrm{Si}$ atom by Ge. To provide insights into the origin of the preferential location of the Ge atoms, we have also examined the location of aluminum (Al) and gallium (Ga) in UTL. In this case each $\mathrm{SiO}_{4}$ was replaced by either an $\mathrm{AlO}_{4}{ }^{-}$or a $\mathrm{GaO}_{4}{ }^{-}$moiety. The total charge of the periodic unit cell was neutralized with a uniform positive background charge. All the calculations in this work were carried out with spin-restricted DFT. All the atoms in the zeolites are all closed-shell systems. Tests with unrestricted DFT gave the same total energies. 


\section{RESULTS AND DISCUSSION}

Physical Properties of UTL and IPC-2a. The optimized unit-cells of silicate UTL and IPC-2a are shown in Figures 1 and 2 respectively. The calculated and experimental structural parameters of these unit cells are presented in Table 1. The largest deviations between the experimental and calculated structural parameters amount to $0.03 \AA$ for the lattice constants and $2.8^{\circ}$ for the lattice angles. This degree of accuracy is not unusual when standard and dispersion-corrected generalized gradient approximation (GGA) functionals are used in periodic DFT optimizations of unit cells. ${ }^{26}$ PBE overestimates the experimental volume by about $1.5-3.4 \%$ while vdW-DF2 predicts slightly larger volumes and optPBE predict slightly smaller volumes, Table 1 . The good performance of the PBE functional relative to the dispersion-corrected vdW-DF2 $2^{19}$, and optPBE ${ }^{20}$ functionals, Table 1, suggest that dispersion effects might not be crucial for obtaining good agreement between calculated and experimental structural properties of these zeolites. Göltl et al. ${ }^{27}$ have shown that the overestimation error of the unit cell volume of a silicate zeolite by

Table 1: Structural parameters of the unit-cells of silicate UTL and IPC-2a.

\begin{tabular}{c|cccc|cccc}
\hline & \multicolumn{4}{|c|}{ UTL } & \multicolumn{4}{c}{ IPC-2a } \\
\hline & PBE & vdW-DF2 & optPBE & Expt. & PBE & vdW-DF2 & optPBE & Expt. $^{4}$ \\
\hline $\mathrm{a}(\AA)$ & 29.5 & 29.6 & 29.5 & 29.80 & 12.5 & 12.6 & 12.5 & 12.27 \\
$\mathrm{~b}(\AA)$ & 14.2 & 14.2 & 14.1 & 13.99 & 13.9 & 13.9 & 13.8 & 14.05 \\
$\mathrm{c}(\AA)$ & 12.6 & 12.6 & 12.5 & 12.39 & 14.0 & 14.0 & 13.9 & 13.82 \\
$\alpha\left({ }^{\circ}\right)$ & 90.0 & 90.0 & 90.0 & 90.0 & 118.6 & 118.5 & 118.4 & 120.3 \\
$\beta\left(^{\circ}\right)$ & 105.4 & 105.4 & 105.4 & 105.2 & 90.3 & 90.3 & 90.3 & 89.9 \\
$\gamma\left({ }^{\circ}\right)$ & 90.0 & 90.0 & 90.0 & 90.0 & 107.1 & 107.1 & 107.1 & 107.0 \\
$\omega\left(\AA^{3}\right)$ & 5059.0 & 5117.6 & 5033.0 & 4987.1 & 2003.7 & 2016.0 & 1995.2 & 1938.2 \\
\hline
\end{tabular}


PBE can be removed by switching to screened hybrid exchange-correlation functionals. Calculations with screened hybrid functionals are however very expensive for UTL and IPC-2a. For this reason, we mainly use the PBE functional in subsequent portions of this manuscript. Similar trends were obtained with the vdW-DF2 and optPBE functionals.

The unit cell volumes of germanate UTL and IPC-2a were calculated as 5724.6 and $2289.8 \AA^{3}$ respectively with the PBE functional. These are about 1.1 times larger than the volumes of their silicate analogues. This volume expansion in the germanate zeolites can be explained by the fact that the covalent radius of Ge is about 1.1 times larger than that of $\mathrm{Si}^{28}$ In a similar vein, the $\mathrm{Si}-\mathrm{O}$ distances in silicate UTL and IPC-2a are about 1.61$1.64 \AA$ in length while the Ge-O distances in their germanate analogues are about 1.77$1.80 \AA$, a ratio of about 1.1. This suggests that the $\mathrm{Si}-\mathrm{O}$ and $\mathrm{Ge}-\mathrm{O}$ bonds are identical with the larger covalent radius of Ge being responsible for the longer bonds and consequently larger unit cell volumes of the germanate zeolites.

Germanosilicate zeolite UTL has 12 unique $\mathrm{T}$ atoms ( $\mathrm{Si}$ or $\mathrm{Ge}$ ) sites. The numerical labels of these sites are shown in Figure 1. The labeling of the unique $\mathrm{T}$ atom positions in Figure 1 is the same as that used by the International Zeolite Association (IZA). ${ }^{29}$ The D4R cages consist of the $\mathrm{T} 1$ and $\mathrm{T} 2$ atoms. The average $\mathrm{O}-\mathrm{Si}-\mathrm{O}$ angles around the atoms in the D4R cages of silicate UTL were calculated to be about $109.3-109.4^{\circ}$ for the $\mathrm{T} 1$ and T2 sites with the PBE functional. These angles are similar to those at other $\mathrm{SiO}_{4}$ sites in UTL. On the other hand, there is some variability in the average Si-O-Si angles centred at the $\mathrm{T}$ atom sites, Figure 4 . The similarity of the $\mathrm{O}-\mathrm{Si}-\mathrm{O}$ angles as well as the variability of the Si-O-Si angles are reproduced by both dispersion-corrected functionals. Regardless of the functional used in the calculations, the D4R cage sites have the lowest average Si-O- 

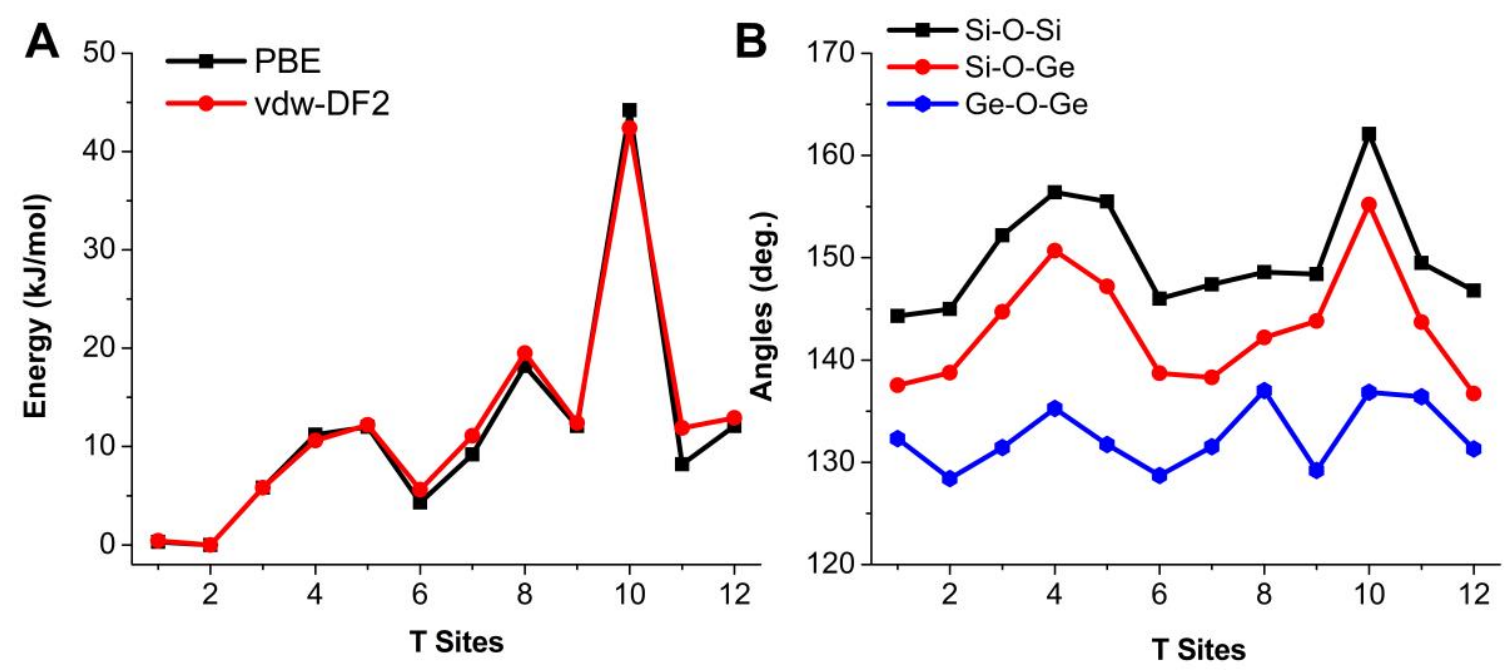

Figure 4: A: The relative energies of the zeolites formed after $\mathrm{Si} / \mathrm{Ge}$ substitutions at various sites in UTL. The results obtained with the PBE (black squares) and vdW-DF2 (red circles) functionals are presented. B: The calculated average Si-O-Si (black squares), $\mathrm{Ge}-\mathrm{O}-\mathrm{Ge}$ (blue hexagons) and $\mathrm{Si}-\mathrm{O}-\mathrm{Ge}$ (red circles) angles centered at the 12 sites in silicate, germanate and germanosilicate UTL obtained at the PBE level of theory.

Si angles. We note that T6, T7, T8, T9 and T12 are also low Si-O-Si centres with angles that are only about $1.6-4.2^{\circ}$ greater than those in the D4R cages, Figure 4 . For germanate UTL, the calculated Ge-O-Ge angles are lower than the Si-O-Si angles in silicate UTL by about $11.6-25.3^{\circ}$. In addition, germanate UTL is distorted such that the site dependence of the T-O-T angles is much more reduced than that found in its silicate version, Figure 4. The optPBE and vdW-DF2 functionals provide similar trends to the calculated Si-O-Si and Ge-O-Ge angles obtained with PBE. The structures optimized with these functionals are provided in the SI. We note that Dawson et al. ${ }^{30}$ have recently used periodic DFT calculations to study the dependence of the total energy on T-O-T angles for several silica and germania zeolites. They found that the broad minimum for Si-O-Si angles is centered around $146^{\circ}$ while the minimum for germania zeolites is centered around $128^{\circ}$, results 
that are consistent with the data presented in Figure 4.

The S4R cages of IPC-2a consist of the T16 and T17 atoms, Figure 2. The average Si-O$\mathrm{Si}$ angles associated with these sites are about $153.0^{\circ}$, Figure 5. These angles are about $10^{\circ}$ larger than those centred at the D4R sites in UTL centred at the D4R sites in UTL, suggesting that the condensation of two S4R units to form a D4R unit in UTL allows for the lower Si-O-Si angles in the latter. In addition, for IPC-2a there are 11 other T-atom sites with average Si-O-Si angles that are lower (by about $1.5-11.2^{\circ}$ ) than those found at T16 and T17.
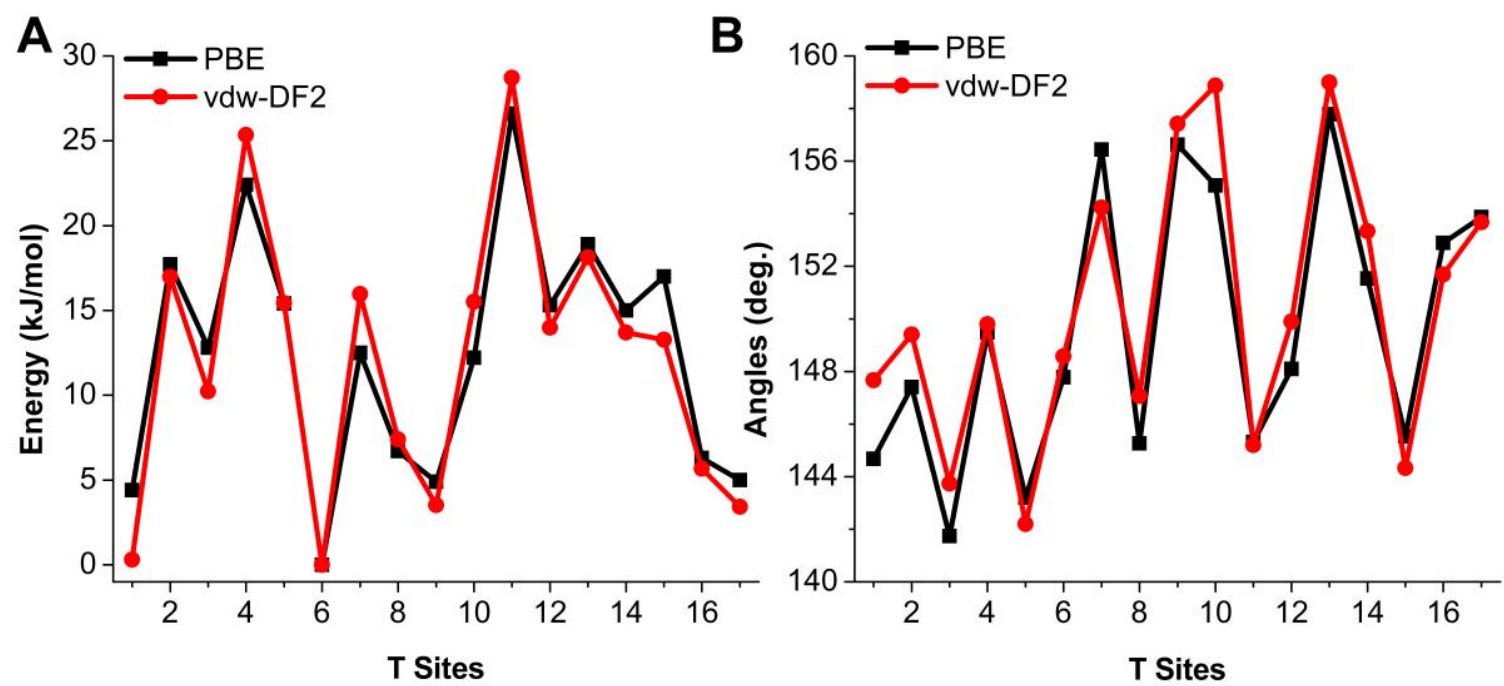

Figure 5: A: The relative energies of the zeolites formed after $\mathrm{Si} / \mathrm{Ge}$ substitutions at various sites in IPC-2a. B: The calculated average Si-O-Si centered at the 17 sites in silicate IPC-2a. The results were obtained with PBE (black square) and vdW-DF2 (red circles).

Ge Substitution in Silicate UTL. The Si/Ge substitution reactions in silicate zeolite UTL are given by (1). The unit cell of UTL is represented as $\mathrm{Si}_{76} \mathrm{O}_{152}$ in this reaction. To calculate the energies associated with preferential siting of Ge at the D4R sites of UTL, we compared the total energies of the zeolites formed by replacing each $\mathrm{Si}$ atom in 
silicate UTL by a Ge atom. In (1), these are represented as $\mathrm{GeSi}_{75} \mathrm{O}_{152}$. The relative energies of these germanosilicate zeolites obtained at the PBE and vdW-DF2 levels of

$$
\mathrm{Si}_{76} \mathrm{O}_{152}(\mathrm{UTL})+\mathrm{Ge}-\mathrm{GeSi}_{75} \mathrm{O}_{152}+\mathrm{Si}
$$

theory are reported in Figure 4. The atomic positions and unit cell volumes of the $\mathrm{GeSi}_{75} \mathrm{O}_{152}$ zeolites were optimized before their total energies were compared. Overall, the trends in the calculated relative energies obtained with PBE are similar to those obtained with the dispersion-corrected vdW-DF2 and optPBE functionals. Si/Ge substitution is most favorable at the T1 and T2 sites found in the D4R cages. These sites are essentially isoenergetic, Table 2, with only an energy difference of about $0.4 \mathrm{~kJ} / \mathrm{mol}$ between them. As previously noted, these sites have the lowest average $\mathrm{Si}-\mathrm{O}-\mathrm{Si}$ angles, suggesting some correlation between this structural property and the preferential location of Ge. ${ }^{11}$

However, of the other sites with low Si-O-Si angles in silicate zeolite UTL, T6, T7, T8, T9 and T12, only T6 is within $6.0 \mathrm{~kJ} / \mathrm{mol}$ of T1 and T2, Figure 4 . The other sites are about 11.1-19.5 kJ/mol less favoured than the D4R sites. Also, although T3 has an average $\mathrm{Si}-\mathrm{O}-\mathrm{Si}$ angle of $152.3^{\circ}$, about $8.0^{\circ}$ higher than the average $\mathrm{Si}-\mathrm{O}-\mathrm{Si}$ angles at the D4R sites, it is only less favourable than T1-T2 for Ge siting by about $5.8 \mathrm{~kJ} / \mathrm{mol}$, Figure 4, and is actually more favourable than T7, T8, T9 and T12. On the other hand, Si/Ge substitution is least favoured at T10, the site with the largest average Si-O-Si angle in UTL. The probability of Ge atoms being located at T10 is very low as a result of the magnitude of the destabilization at this site, $42.4 \mathrm{~kJ} / \mathrm{mol}$ in Figure 4 , relative to the D4R sites. In any case, this site is embedded deeply in the zeolite framework and would not be in contact with $\mathrm{Ge}$ reagents during experimental $\mathrm{Si} / \mathrm{Ge}$ substitutions. All these results 
suggest that the preferential siting of Ge in UTL appears to at least strongly favour sites with low average $\mathrm{Si}-\mathrm{O}-\mathrm{Si}$ angles. The $\mathrm{Si}-\mathrm{O}-\mathrm{Ge}$ angles formed after the $\mathrm{Si} / \mathrm{Ge}$ substitutions follow the same trend as the Si-O-Si angles in silicate UTL, Figure 4. It is probably the case that the $\mathrm{Si}-\mathrm{O}-\mathrm{Si}$ angles in silicate UTL act as a template for the low SiO-Ge angles that would be favourable sites for locating Ge atoms. In addition, there is an increase in the unit cell volume of zeolite UTL after Si/Ge substitutions. Sites with lower $\mathrm{Si}-\mathrm{O}-\mathrm{Si}$ angles in UTL and $\mathrm{Si}-\mathrm{O}-\mathrm{Ge}$ angles after the $\mathrm{Si} / \mathrm{Ge}$ substitutions do not necessarily result in the smallest expansion of the unit cell, Figure 4. There therefore

Table 2: The relative energies and associated volume changes for $\mathrm{Si} / \mathrm{Ge}, \mathrm{Si} / \mathrm{Al}^{-}$and $\mathrm{Si} / \mathrm{Ga}^{-}$substitutions at each unique $\mathrm{Si}$ site. These data were obtained at the PBE level of theory except where noted. The absolute energies for inserting a Ge atom at T2 are 455.0 and $463 \mathrm{~kJ} / \mathrm{mol}$ at the PBE and optPBE levels respectively.

\begin{tabular}{r|cccc|cccc}
\hline & \multicolumn{3}{|c|}{ Relative Energies (kJ/mol) } & \multicolumn{4}{c}{ Volume Change $\left(\AA^{3}\right)$} \\
\hline & Si/Ge & Si/Ge;optPBE & Si/Al- & Si/Ga & Si/Ge & Si/Ge;optPBE & Si/Al $^{-}$ & Si/Ga $^{-}$ \\
\hline T1 & 0.4 & 0.3 & 5.9 & 4.0 & 18.7 & 21.0 & 29.4 & 33.6 \\
T2 & 0.0 & 0.0 & 2.7 & 0.0 & 20.0 & 21.4 & 30.9 & 33.6 \\
T3 & 5.8 & 5.8 & 4.9 & 8.3 & 21.1 & 19.2 & 22.6 & 36.3 \\
T4 & 10.6 & 11.2 & 0.0 & 9.4 & 21.8 & 25.6 & 31.2 & 43.0 \\
T5 & 12.2 & 12.1 & 10.0 & 19.8 & 4.4 & 4.7 & 17.3 & 28.6 \\
T6 & 5.6 & 4.1 & 9.8 & 6.9 & 9.3 & 9.9 & 28.2 & 33.3 \\
T7 & 11.1 & 9.9 & 17.1 & 19.8 & 5.4 & 8.5 & 29.8 & 31.2 \\
T8 & 19.5 & 18.3 & 23.3 & 36.2 & 12.1 & 11.0 & 28.6 & 39.1 \\
T9 & 12.4 & 11.7 & 15.3 & 22.6 & 7.9 & 7.5 & 20.2 & 25.9 \\
T10 & 42.4 & 43.9 & 23.5 & 58.1 & 3.3 & 7.4 & 12.0 & 14.4 \\
T11 & 11.9 & 8.8 & 8.3 & 19.1 & 6.4 & 2.2 & 18.2 & 26.5 \\
T12 & 12.9 & 8.2 & 22.4 & 26.9 & -4.0 & -2.1 & 15.9 & 12.4 \\
\hline
\end{tabular}


appears to be little correlation between the volume increase after $\mathrm{Si} / \mathrm{Ge}$ substitution and preferential siting at the $\mathrm{D} 4 \mathrm{R}$ sites.

The relative energies of the structures formed after replacing the $\mathrm{SiO}_{4}$ group at each site in silicate $\mathrm{UTL}$ by $\mathrm{AlO}_{4}{ }^{-}$( $\mathrm{Si} / \mathrm{Al}^{-}$substitution) and $\mathrm{GaO}_{4}{ }^{-}\left(\mathrm{Si} / \mathrm{Ga}^{-}\right.$substitution) are also presented in Table 2. For Si/ $\mathrm{Ga}^{-}$substitutions, the D4R sites $(0.0 \mathrm{~kJ} / \mathrm{mol}$ for $\mathrm{T} 2$ and 4.0 $\mathrm{kJ} / \mathrm{mol}$ for $\mathrm{T} 1$ ) are preferentially preferred, just like in the case of the $\mathrm{Si} / \mathrm{Ge}$ substitutions. In contrast, the $\mathrm{T} 4$ site is the most favourable for $\mathrm{Si}^{-\mathrm{Al}^{-}}$substitution. As shown in Figure 1 , the $\mathrm{T} 4$ site is one of the layer sites lining the 2D pores/channels running through UTL. This confirms the experimental work of Shamzy et al. ${ }^{31}$ who found that $\mathrm{Al}$ is localized in the layers of UTL in contrast to the preferential localization of Ge in the D4R cages. The preferential localization of $\mathrm{Al}$ at the $\mathrm{T} 4$ layer sites implies that $\mathrm{Si} / \mathrm{Al}^{-}$substitutions can occur during exposure to reagents. However, the ADOR process (which requires localization at the D4R sites) cannot be initiated by loading $\mathrm{Al}$ into silicate UTL. We note that during $\mathrm{Si} / \mathrm{Al}^{-}$substitutions, the $\mathrm{D} 4 \mathrm{R}$ sites are only $2.7-4.0 \mathrm{~kJ} / \mathrm{mol}$ less favourable than at T4. Overall, the preference for siting at the D4R sites appears to follow the trend, $\mathrm{Si} / \mathrm{Al}^{-}<\mathrm{Si} / \mathrm{Ge}<\mathrm{Si} / \mathrm{Ga}^{-}$, Table 2 . This is in concordance with the trend in the empirical covalent radii of the substitute elements, $1.18 \AA$ for $\mathrm{Al}, 1.22 \AA$ for $\mathrm{Ge}$ and $1.26 \AA$ for $\mathrm{Ga}^{28}{ }^{28}$ Compared to $\mathrm{Si}$ and $\mathrm{Al}$, the larger elements, Ge and Ga, would require smaller T-O$\mathrm{T}$ angles, which in silicate UTL are provided at the T atoms in the D4R cages.

We note that the $\mathrm{Si} / \mathrm{Al}^{-}, \mathrm{Si} / \mathrm{Ge}$ and $\mathrm{Si} / \mathrm{Ga}^{-}$substitutions are all endothermic reactions. We have only presented the relative energies of the resulting zeolites in Figures 4 and 5 as well as in Table 2. These relative energies also depict the relative endothermicity of substitution at each site. 
Propagation of D4R Preference during Si/Ge Substitutions in UTL. As shown in Figure 4 and Table 2, the zeolites formed by performing Si/Ge substitutions at the D4R sites (T1 and T2) are only 5.8 and $5.6 \mathrm{~kJ} / \mathrm{mol}$ more stable than those formed by carrying out substitutions at T3 and T6 respectively. The magnitudes of these energies would suggest that some Ge atoms might be sited at T3 and T6 rather than exclusively at T1 and T2. In order to provide further insights into how Ge siting at the D4R sites is propagated under experimental conditions, we have also computed the relative energies of locating a second $\mathrm{Ge}$ atom at other $\mathrm{T}$ atom sites after one $\mathrm{Ge}$ atom has been located at T1, T2, T3 or T6, Figure 6. These four sites were selected as they are the most energetically favoured for locating one and only one Ge atom in UTL, Figure 4. For UTL with one Ge atom already at the $\mathrm{T} 1$ site, insertion of a second Ge atom is most favoured at T2. Also for UTL with one Ge atom already at a T2 site, the insertion of a second Ge atom is most favoured at T1. For UTL with one Ge atom at T3 or T6, insertion of a second Ge atom at the $\mathrm{T} 1$ and $\mathrm{T} 2$ sites are at least $7.0-7.4 \mathrm{~kJ} / \mathrm{mol}$ more favourable than any other $\mathrm{T}$ atom site. These results suggest that, irrespective of the location of the first Ge atom in germanosilicate UTL, the D4R sites remain the most favoured sites for locating a second Ge atom. The substitution energies required to locate a second Ge atom at $\mathrm{T} 1$ or T2 if there is already one Ge atom at T1, T2, T3 or T6 are shown in Table 3. Compared to the results obtained after only one $\mathrm{Si} / \mathrm{Ge}$ substitution, Table 2, the D4R sites are much more energetically preferred than the T3 and T6 sites, when two $\mathrm{Si}$ atoms are replaced by Ge atoms in silicate UTL. This appears to be a cooperative effect and suggests that Ge atoms would become increasingly concentrated at the D4R sites of UTL as more Si/Ge substitutions are performed. 


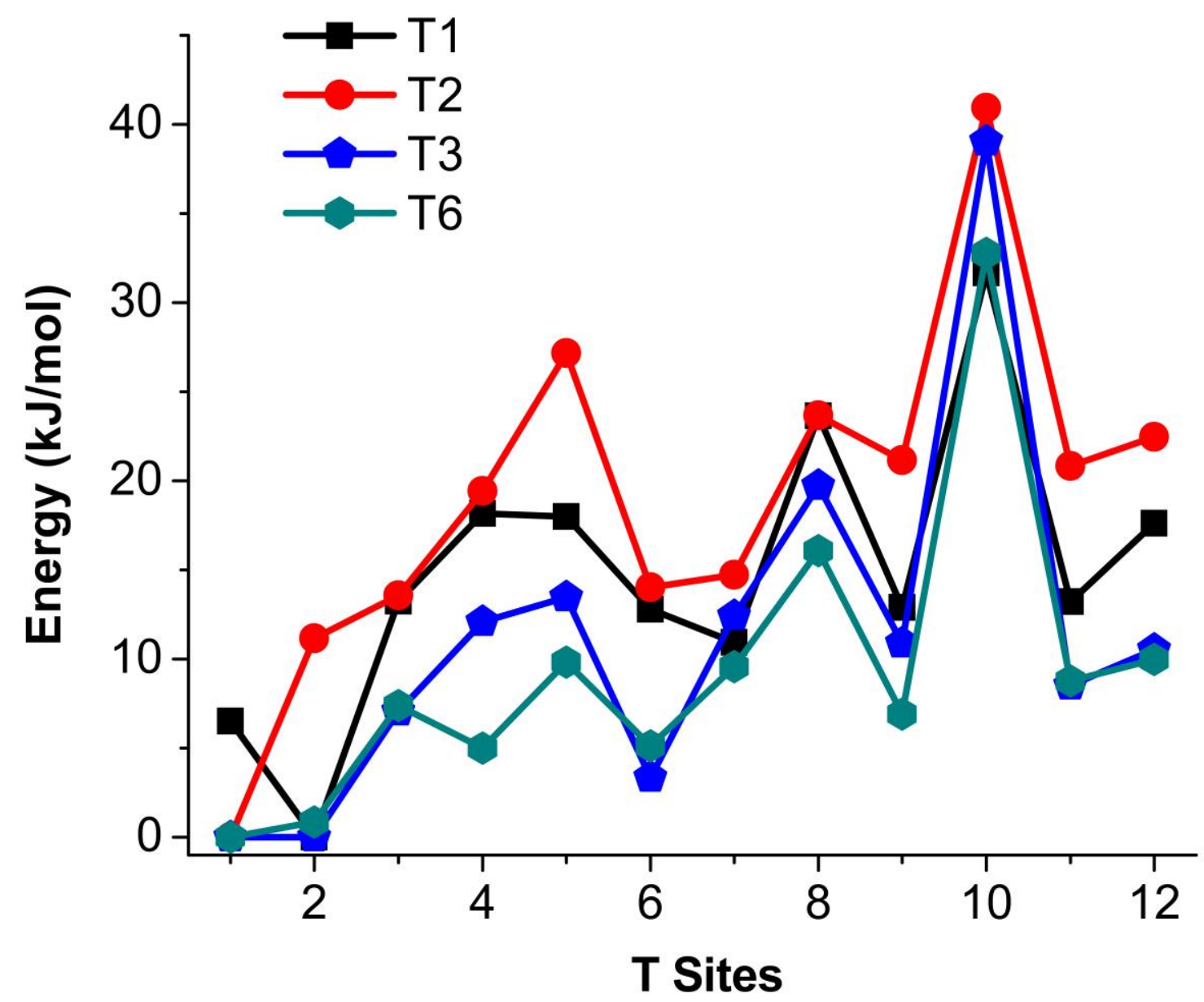

Figure 6: The relative energies of the various germanosilicate zeolites formed after a second $\mathrm{Si} / \mathrm{Ge}$ substitution in UTL that already contains a Ge atom at T1 (black squares), T2 (red circles), T3 (blue pentagons) and T6 (green hexagons).

Another way of looking at the cooperative effect is by actually computing the reaction energy of reaction (2). In this reaction, one Ge atom is present at any of T1, T2, T3 or T6

$$
\mathrm{Si}_{76} \mathrm{O}_{152}(\mathrm{UTL})+2 \mathrm{Ge}-\mathrm{Ge}_{2} \mathrm{Si}_{74} \mathrm{O}_{152}+2 \mathrm{Si}
$$

and a second Ge atom is placed at either T1 or T2. The electronic atomic energies Si and Ge were used to compute the energies that are presented in Table 3. Zero point energy corrections $(\mathrm{ZPE})$ were added to the reaction electronic energies, $\Delta \mathrm{E}$. The most favoured configuration for double $\mathrm{Si} / \mathrm{Ge}$ substitution is $\mathrm{T} 1 \mathrm{~T} 2$ ( $\mathrm{Ge}$ atoms at $\mathrm{T} 1$ and $\mathrm{T} 2$ ). The 
zeolites containing one Ge atom at T3 and T6 are about $13-14 \mathrm{~kJ} / \mathrm{mol}$ less stable than the T1T2 configuration. This suggest to us that the T3T1, T6T1, T3T2, T6T2 configurations as well as other configurations containing Ge atoms outside the D4R cages will exist only in minor quantities under ambient conditions. In addition, we found that for T1T2, the relative energies for an additional (the third) Si/Ge substitution at the T1, T2, T3, T4, T5, T6, T7, T8, T9, T10, T11 and T12 sites are 7.1, 0.0, 13.4, 26.6, 18.5, 18.9, 17.4, 24.3, 22.1, 36.5, 22.3 and $19.7 \mathrm{~kJ} / \mathrm{mol}$ respectively. This indicates that at least three Ge atoms would have to be incorporated into the $\mathrm{D} 4 \mathrm{R}$ units before there can be any appreciable occupancy at the other $\mathrm{T}$ atom sites. Simply put, increasing Ge/Si ratios will lead to more Ge atoms in the D4R cages.

Table 3: The energy $(\mathrm{kJ} / \mathrm{mol})$ for $\mathrm{Si} / \mathrm{Ge}$ substitution the $\mathrm{T} 2$ site when a $\mathrm{Ge}$ atom is already present at one of T1 (T1T2 configuration) is $927.0 \mathrm{~kJ} / \mathrm{mol}$. The relative energies of other Si/Ge zeolites involving the T1, T2, T3 and T6 sites are given relative to T1T2.

\begin{tabular}{c|cccc}
\hline & T1 & T2 & T3 & T6 \\
\hline T1 & 6.9 & 0.0 & 13.6 & 13.1 \\
T2 & 0.3 & 11.2 & 13.6 & 14.0 \\
\hline
\end{tabular}

\section{Ge Substitution in Silicate IPC-2a}

We have also studied Ge localization in IPC-2a to determine whether its S4R cages represent preferential sites, just as the D4R cages in UTL. The relative energies of the germanosilicate zeolites formed after Si/Ge substitution in silicate IPC-2a were calculated in a similar manner as that used for UTL. These energies are reported in Figure 5. Substitution is most favoured at T6, which is found in the layers but is exposed to the pore channels running through the zeolite, Figure 2. Other atoms lining the channels that 
favour Ge substitution are $\mathrm{T} 1, \mathrm{~T} 8$ and $\mathrm{T} 9 . \mathrm{Si} / \mathrm{Ge}$ substitutions at these sites are generally within 4.4-6.7 kJ/mol of the $\mathrm{T} 6$ site. Although Ge atoms at the layer $\mathrm{T}$ positions around the pore channels can be leached out, the preferential localization at these sites in IPC-2a precludes the possibility of exactly reversing the ADOR process (building UTL from IPC-2a) via condensation of Ge-rich portions of the framework as well as the possibility of condensing the S4R cages into the oxygen linkers found in IPC-4. This is because the T16 and T17 sites found in the S4R cages of IPC-2a are about 5.0-6.3 kJ/mol less favourable than the T6 site. Simply put, if the S4R rings cannot be leached out, then they cannot be condensed to form D4R cages or replaced by oxygen atoms. These inferences agree well with the experimental procedures used by Čejka et al. ${ }^{4}$ in preparing IPC-2a and IPC-4 from UTL. Overall, the results obtained with PBE agree well with those obtained with the dispersion-corrected functionals, Figure 5.

From a perspective of relevance to experimental investigations, although delamination of the Ge atoms in the D4R sites of UTL would by necessity result in the presence of Ge atoms in the S4R sites of IPC-2a, our results (Figure 5) suggest that the Ge atoms will have to 'migrate' to other sites or exit the zeolite, as a result of the energy penalty associated with these sites relative to other sites such as T1 and T6. Overall, the nature of the leaching process will determine the proportion of Ge atoms that are removed from the D4R groups (de-intercalation) as well as the amount of re-organization that would have to occur to remove (or migrate out) Ge atoms from the S4R sites. This suggests to us that the ADOR process involves significant reorganization and de-intercalation of the silica framework. Indeed, Wheatley et al. ${ }^{32}$ have been able to use continuous control of the relative rates of these processes to synthesize several zeolites with continuously tuneable 
surface area and pore volumes. Also, in their study of the inverse $\sigma$ transformation of the UTL zeolite to all-silica COK-14, Verheyen et al. ${ }^{33}$ were able to isolate an intermediate state in which dislodged germanate four-rings were found in the pore channels.

Unlike in UTL, it is somewhat difficult to draw any direct correlations between the average $\mathrm{Si}-\mathrm{O}-\mathrm{Si}$ angles at each $\mathrm{T}$ atom site and the preferential location of Ge in IPC-2a, Figure 5. This suggests that the structural factors that determine the most favourable sites for Ge atoms might differ for various zeolites. There are however several similarities in the trends seen in the preference for Ge atom location in IPC-2a and UTL. Firstly, Si/Ge substitution is least favoured at T10 in UTL, Figure 1, and at T11 in IPC-2a, Figure 2. These sites are both embedded in the zeolite framework and are likely inaccessible to Ge reagents during $\mathrm{Si} / \mathrm{Ge}$ substitutions. Secondly, there is very little correlation between the expansion of the unit cell and the relative energies of the germanosilicate zeolites formed after Si/Ge substitution. Thirdly, the preference for Ge atoms at certain sites (D4R sites in UTL, Figure 6 and Table 3), and layer sites around the pore channels in IPC-2a atoms, (Figure 1 of SI) increase as more Si atoms are replaced by Ge.

\section{Conclusions}

We have studied the preferential location of Ge atoms in the D4R cages of the UTL zeolite using periodic DFT calculations with standard and dispersion-corrected GGA density functionals. In addition, we compared the localization of Ge in this zeolite to that of $\mathrm{Al}$ and $\mathrm{Ga}$. The generality of the T-O-T angles as a determinant for Ge atom site preferences was examined by comparing UTL to its derivative, IPC-2a, which has S4R cages. Our results can be summarized as follows: 
1. Ge atoms are preferentially located in the D4R cages of UTL. These sites have the lowest average T-O-T angles in the zeolite and are favored by $5.6-42.4 \mathrm{~kJ} / \mathrm{mol}$ relative to other $\mathrm{T}$ atom sites.

2. Our calculations suggest that at least $3 \mathrm{Ge}$ atoms will be located in the D4R sites before any appreciable occupancy of the other sites. The D4R sites are more preferred to other $\mathrm{T}$ atoms sites after double or triple $\mathrm{Si} / \mathrm{Ge}$ substitutions than when only one $\mathrm{Si}$ atom is changed to $\mathrm{Ge}$, suggesting a cooperative effect. As more $\mathrm{Si}$ atoms are replaced with Ge atoms, the preference for localization in the D4R cages is increased.

3. Like Ge, Ga is preferentially located in the D4R cages of UTL whereas Al prefers the layers around the pore channels. This indicates that larger atoms, Ga and $\mathrm{Ge}$, prefer the lower T-O-T angles found in the D4R cages whereas smaller atoms, $\mathrm{Al}$ and $\mathrm{Si}$, prefer to arrange into polyhedra with higher T-O-T angles. The localization of $\mathrm{Al}$ in the layer sites around the pore channels confirm previously reported experimental data.

4. Ge atoms are preferentially located at the T6 site in IPC-2a, a layer site around the pores channels of this zeolite. This indicates that while Ge atoms can be leached out in IPC-2a, they cannot however participate in the ADOR process as they are not located in the S4R cages. The S4R cages of IPC-2a can therefore not be condensed into $\mathrm{D} 4 \mathrm{R}$ cages or replaced by oxygen atoms. Indeed, the localization of $\mathrm{Ge}$ in the layers around the 2D channels of IPC-2a implies that this zeolite is a terminal product of the ADOR process. 
5. In contrast to UTL where there is some correlation between the preference for Ge at certain sites and the average $\mathrm{Si}-\mathrm{O}-\mathrm{Si}$ angles centered at those sites, such correlations are difficult to see in IPC-2a. This suggests that the factors determining the preferential location of Ge atoms might differ for zeolites that do not have D4R cages.

6. In addition, comparison of the energy required to substitute a Si atom in UTL and IPC-2a by Ge shows that substitution at the best sites in UTL (T1 and T2) is about 2 $\mathrm{kJ} / \mathrm{mol}$ more favorable than the best site in IPC-2a (T1 and T6). This further reinforces the fact that Ge will not be found at the S4R sites of IPC-2a.

7. The periodic DFT results presented in this study provide some insights into the ADOR process that leads from the UTL zeolite to IPC-2a and IPC-4. DFT calculations with cluster models and force field simulations with model potentials are most likely unable to fully describe the preferential localization of atoms in zeolites, as a result of unphysical edge effects, spatial anisotropy in cluster models and possible dependence of the $\mathrm{Si} / \mathrm{Ge}$ (or replacement of $\mathrm{Si}$ atoms by any other metal) replacement potential on subtle chemical changes at different $\mathrm{T}$ atom sites, a factor missing from force field simulations. The implication of our work is that periodic DFT calculations with standard and dispersion-corrected functionals can be used to, a priori, determine the final products of reactions involving leaching or insertion of specific atoms into zeolites. These reactions are very important in continuing attempts to synthesize and characterize rationally designed zeolites.

\section{AUTHOR INFORMATION}

\section{Corresponding Authors}


gagliard@umn.edu, mwdeem@ rice.edu

\section{ACKNOWLEDGEMENTS}

This work was supported through the Nanoporous Materials Genome Center of the

US Department of Energy, Office of Basic Energy Sciences, Division of Chemical

Sciences, Geosciences and Biosciences under Award Number DE-FG0212ER16362.

\section{ASSOCIATED CONTENT}

\section{Supporting Information}

Some optimized structures obtained from periodic DFT calculations. Relative energies zeolites formed after $\mathrm{Si} / \mathrm{Ge}$ substitutions at various sites in IPC-2a that already contains a Ge atom at T6. This material is available free of charge via the Internet at http://pubs.acs.org.

\section{REFERENCES}

1. www.iza-structure.org/databases/books/Atlas 6ed.pdf.

2. Corma, A.; Diaz-Cabanas, M. J.; Rey, F.; Nicolooulas, S.; Boulahya, K., ITQ-15: The first ultralarge pore zeolite with a bi-directional pore system formed by intersecting 14- and 12-ring channels, and its catalytic implications. Chem. Commun. 2004, (12), 1356-1357.

3. Paillaud, J. L.; Harbuzaru, B.; Patarin, J.; Bats, N., Extra-large-pore zeolites with two-dimensional channels formed by 14 and 12 rings. Science 2004, 304 (5673), 990-992.

4. $\quad$ Roth, W. J.; Nachtigall, P.; Morris, R. E.; Wheatley, P. S.; Seymour, V. R.; Ashbrook, S. E.; Chlubna, P.; Grajciar, L.; Polozij, M.; Zukal, A.; Shvets, O.; Cejka, J., A family of zeolites with controlled pore size prepared using a top-down method. Nat. Chem. 2013, 5 (7), 628-633. 
5. $\quad$ Corma, A.; Navarro, M. T.; Rey, F.; Rius, J.; Valencia, S., Pure polymorph C of zeolite beta synthesized by using framework isomorphous substitution as a structure-directing mechanism. Angew. Chem. Int. Edit. 2001, 40 (12), 2277-+.

6. (a) Corma, A.; Diaz-Cabanas, M.; Martinez-Triguero, J.; Rey, F.; Rius, J., A largecavity zeolite with wide pore windows and potential as an oil refining catalyst. Nature 2002, 418 (6897), 514-517; (b) Corma, A.; Diaz-Cabanas, M. J.; Rey, F., Synthesis of ITQ-21 in OH- media. Chem. Commun. 2003, (9), 1050-1051; (c) Corma, V.; Diaz-Cabanas, M. J.; Fornes, V., Synthesis, characterization, and catalytic activity of a large-pore tridirectional zeolite, H-ITQ-7. Angew. Chem. Int. Edit. 2000, 39 (13), 2346-2349.

7. (a) Roth, W. J.; Nachtigall, P.; Morris, R. E.; Cejka, J., Two-Dimensional Zeolites: Current Status and Perspectives. Chemical Reviews 2014, 114 (9), 48074837; (b) Roth, W. J.; Shvets, O. V.; Shamzhy, M.; Chlubna, P.; Kubu, M.; Nachtigall, P.; Cejka, J., Postsynthesis Transformation of Three-Dimensional Framework into a Lamellar Zeolite with Modifiable Architecture. J. Am. Chem. Soc. 2011, 133 (16), 6130-6133.

8. McCusker, L. B.; GrosseKunstleve, R. W.; Baerlocher, C.; Yoshikawa, M.; Davis, M. E., Synthesis optimization and structure analysis of the zincosilicate molecular sieve VPI-9. Microporous Mater. 1996, 6 (5-6), 295-309.

9. $\quad$ Freyhardt, C. C.; Lobo, R. F.; Khodabandeh, S.; Lewis, J. E.; Tsapatsis, M.; Yoshikawa, M.; Camblor, M. A.; Pan, M.; Helmkamp, M. M.; Zones, S. I.; Davis, M. E., VPI-8: A high-silica molecular sieve with a novel "pinwheel" building unit and its implications for the synthesis of extra-large pore molecular sieves. J. Am. Chem. Soc. 1996, 118 (31), 7299-7310.

10. O'Keeffe, M.; Yaghi, O. M., Germanate zeolites: Contrasting the behavior of germanate and silicate structures built from cubic T8020 units ( $\mathrm{T}=\mathrm{Ge}$ or Si. ChemEur. J. 1999, 5 (10), 2796-2801.

11. Sastre, G.; Pulido, A.; Corma, A., An attempt to predict and rationalize relative stabilities and preferential germanium location in Si/Ge zeolites. Micropor. Mesopor. Mat. 2005, 82 (1-2), 159-163.

12. Zwijnenburg, M. A.; Bromley, S. T.; Jansen, J. C.; Maschmeyer, T., Computational insights into the role of $\mathrm{Ge}$ in stabilising double-four ring containing zeolites. Micropor. Mesopor. Mat. 2004, 73 (3), 171-174.

13. Kamakoti, P.; Barckholtz, T. A., Role of germanium in the formation of double four rings in zeolites. J. Phys. Chem. C 2007, 111 (9), 3575-3583.

14. (a) Conradsson, T.; Dadachov, M. S.; Zou, X. D., Synthesis and structure of (Me3N)(6) Ge32064 center $\operatorname{dot}(\mathrm{H} 20)(4.5)$, a thermally stable novel zeotype with 3D interconnected 12-ring channels. Micropor. Mesopor. Mat. 2000, 41 (1-3), 183-191; (b) Liu, Z.; Ohsuna, T.; Terasaki, O.; Camblor, M. A.; Diaz-Cabanas, M. J.; Hiraga, K., The first zeolite with three-dimensional intersecting straight-channel system of 12membered rings. J. Am. Chem. Soc. 2001, 123 (22), 5370-5371.

15. (a) Kresse, G.; Furthmuller, J., Efficient iterative schemes for ab initio totalenergy calculations using a plane-wave basis set. Phys. Rev. B 1996, 54 (16), 1116911186; (b) Kresse, G.; Furthmuller, J., Efficiency of ab-initio total energy calculations for metals and semiconductors using a plane-wave basis set. Comp. Mater. Sci. 1996, $6(1), 15-50$. 
16. Blochl, P. E., PROJECTOR AUGMENTED-WAVE METHOD. Phys. Rev. B 1994, $50(24), 17953-17979$.

17. Perdew, J. P.; Burke, K.; Ernzerhof, M., Generalized gradient approximation made simple. Phys. Rev. Lett. 1996, 77 (18), 3865-3868.

18. Kristyan, S.; Pulay, P., Can (semi)local density-functional theory account for the London dispersion forces. Chem. Phys. Lett. 1994, 229 (3), 175-180.

19. Lee, K.; Murray, E. D.; Kong, L.; Lundqvist, B. I.; Langreth, D. C., Higheraccuracy van der Waals density functional. Phys. Rev. B 2010, 82 (8).

20. (a) Klimes, J.; Bowler, D. R.; Michaelides, A., Chemical accuracy for the van der Waals density functional. J. Phys-Condes. Mat. 2010, 22 (2); (b) Klimes, J.; Bowler, D. R.; Michaelides, A., Van der Waals density functionals applied to solids. Phys. Rev. $B$ 2011, 83 (19).

21. Heany, P. J.; Prewitt, C. T.; Gibbs, G. V., Physical Behavior, Geochemistry and Materials Applications. Mineralogical Society of America: Washington, D.C., 1994.

22. Petrovic, I.; Heaney, P. J.; Navrotsky, A., Thermochemistry of the new silica polymorph moganite. Physics and Chemistry of Minerals 1996, 23 (2), 119-126.

23. Saxena, S. K.; Chatterjee, N.; Fei, Y.; Shen, G., Thermodynamic Data on Oxides and Silicates. Springer-Verlag: Berlin, 1993.

24. Catti, M.; Civalleri, B.; Ugliengo, P., Structure and energetics of SiO2 polymorphs by quantum-mechanical and semiclassical approaches. Journal of Physical Chemistry B 2000, 104 (31), 7259-7265.

25. Perdew, J. P.; Zunger, A., Self-interaction correction to density-functional approximations for many-electron systems. Phys. Rev. B 1981, 23, 5048-5079.

26. Kurth, S.; Perdew, J. P.; Blaha, P., Molecular and solid-state tests of density functional approximations: LSD, GGAs, and meta-GGAs. Int. J. Quantum Chem. 1999, 75 (4-5), 889-909.

27. Goltl, F.; Hafner, J., Structure and properties of metal-exchanged zeolites studied using gradient-corrected and hybrid functionals. I. Structure and energetics. Journal of Chemical Physics 2012, 136 (6).

28. Huheey, J. E.; Keiter, E. A.; Keiter, R. L., Inorganic Chemistry: Principles of Structure and Reactivity. HarperCollins: New York, USA, 1993.

29. www.iza-structure.org/databases.

30. Dawson, C. J.; Sanchez-Smith, R.; Rez, P.; O'Keeffe, M.; Treacy, M. M. J., Ab Initio Calculations of the Energy Dependence of Si-O-Si Angles in Silica and Ge-O-Ge Angles in Germania Crystalline Systems. Chemistry of Materials 2014, 26 (4), 15231527.

31. Shamzhy, M. V.; Shvets, O. V.; Opanasenko, M. V.; Yaremov, P. S.; Sarkisyan, L. G.; Chlubna, P.; Zukal, A.; Marthala, V. R.; Hartmann, M.; Cejka, J., Synthesis of isomorphously substituted extra-large pore UTL zeolites. J. Mater. Chem. 2012, 22 (31), 15793-15803.

32. Wheatley, P. S.; Chlubná-Eliášová, P.; Greer, H.; Zhou, W.; Seymour, V. R.; Dawson, D. M.; Ashbrook, S. E.; Pinar, A. B.; McCusker, L. B.; Opanasenko, M.; Čejka, J.; Morris, R. E., Zeolites with Continuously Tuneable Porosity. Angewandte Chemie 2014, n/a-n/a.

33. Verheyen, E.; Joos, L.; Van Havenbergh, K.; Breynaert, E.; Kasian, N.; Gobechiya, E.; Houthoofd, K.; Martineau, C.; Hinterstein, M.; Taulelle, F.; Van 
Speybroeck, V.; Waroquier, M.; Bals, S.; Van Tendeloo, G.; Kirschhock, C. E. A.; Martens, J. A., Design of zeolite by inverse sigma transformation. Nature Materials 2012, 11 (12), 1059-1064.

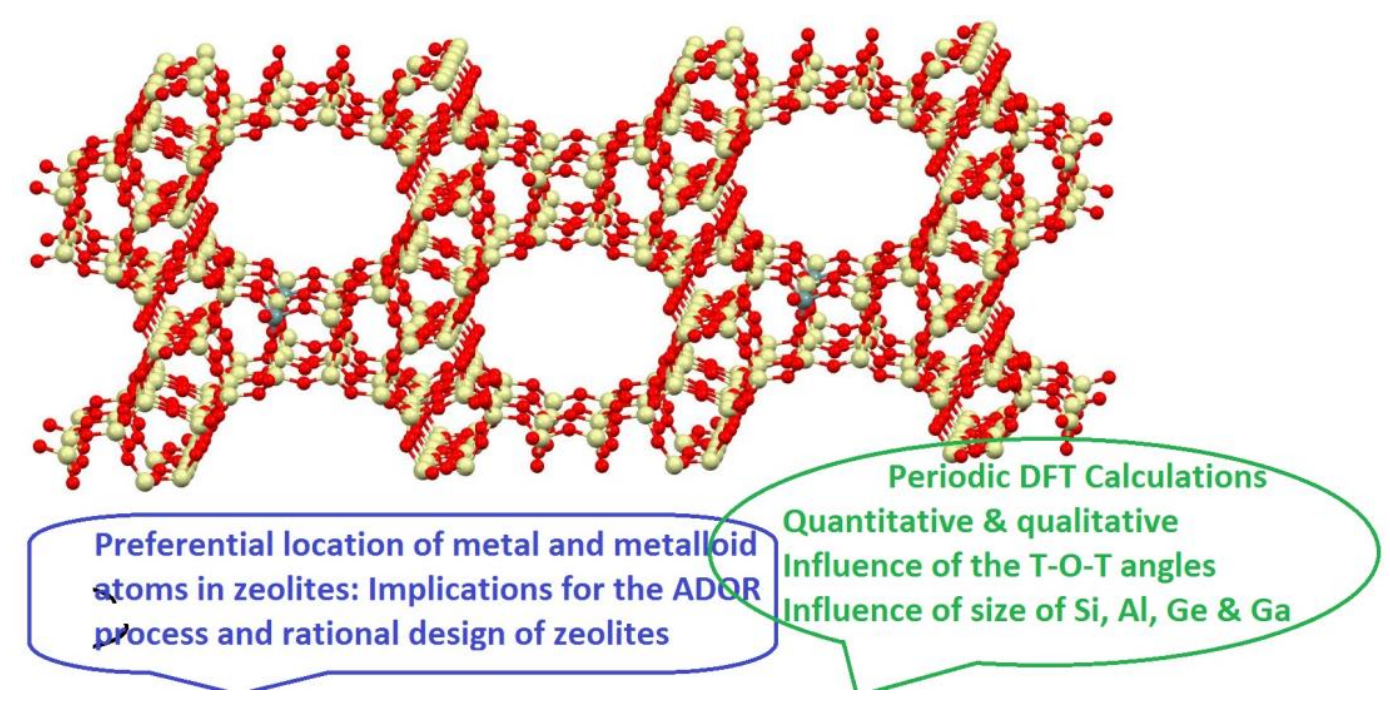

\section{SYNOPSIS TABLE OF CONTENT}

Periodic density functional theory calculations show that Ge atoms are preferentially sited at the T1 and T2 atoms in the D4R cages of zeolite UTL. The preference for these D4R sites over other sites increases as more Si atoms are replaced by Ge. The ADOR process of zeolite synthesis by Ge leaching and solid annealing relies on this localization of Ge. 